\title{
Differences in cortical response to acupressure and electroacupuncture stimuli
}

\author{
Thomas Witzel ${ }^{1}$, Vitaly Napadow ${ }^{1,2}$, Norman W Kettner ${ }^{2}$, Mark G Vangel ${ }^{1}$, Matti S Hämäläinen ${ }^{1}$ and \\ Rupali P Dhond ${ }^{1 *}$
}

\begin{abstract}
Background: FMRI studies focus on sub-cortical effects of acupuncture stimuli. The purpose of this study was to assess changes in primary somatosensory (S1) activity over the course of different types of acupuncture stimulation. We used whole head magnetoencephalography (MEG) to map S1 brain response during 15 minutes of electroacupuncture (EA) and acupressure (AP). We further assessed how brain response changed during the course of stimulation.

Results: Evoked brain response to EA differed from AP in its temporal dynamics by showing clear contralateral M20/M30 peaks while the latter demonstrated temporal dispersion. Both EA and AP demonstrated significantly decreased response amplitudes following five minutes of stimulation. However, the latency of these decreases were earlier in EA ( 30 ms post-stimulus) than AP (> $100 \mathrm{~ms}$ ). Time-frequency responses demonstrated early onset, event related synchronization (ERS), within the gamma band at 70-130 ms and the theta band at $\sim 50-200 \mathrm{~ms}$ post-stimulus. A prolonged event related desynchronization (ERD) of alpha and beta power occurred at $\sim 100-300$ ms post-stimulus. There was decreased beta ERD at 100-300 ms over the course of EA, but not AP.
\end{abstract}

Conclusion: Both EA and AP demonstrated conditioning of SI response. In conjunction with their subcortical effects on endogenous pain regulation, these therapies show potential for affecting S1 processing and possibly altering maladaptive neuroplasticity. Thus, further investigation in neuropathic populations is needed.

\section{Background}

Acupuncture is a component of Traditional Chinese Medicine (TCM) most often characterized by stimulation of specific body sites with sharp 'insertive' needles. However, blunt 'non-insertive' needles (Teishein needles) may also be used to apply rapid percussive pressure, specifically tapping, without penetrating the skin, akin to 'acupressure'. Modern insertive acupuncture frequently involves electrical stimulation applied directly to inserted needles (electroacupuncture). In a typical clinical acupuncture session, a patient may be stimulated manually or electrically at one or more body sites for > 10 minutes. During this time the patient is often left alone to 'relax' without additional sensory stimuli and there is no requirement for focused attention. Recently, acupuncture has been gaining popularity in the West as

\footnotetext{
* Correspondence: polly@nmr.mgh.harvard.edu

'Harvard Medical School, Martinos Center for Biomedical Imaging,

Charlestown, MA 02129 USA

Full list of author information is available at the end of the article
}

a complementary therapy and much research is aimed at elucidating its neural correlates.

Currently, fMRI data show that insertive acupuncture activates subcortical brain areas which are implicated in endogenous anti-nociception [for review 1]. Numerous animal studies show that acupuncture analgesia is mediated by opiodergic and/or monoaminergic neurotransmission involving the brainstem, thalamus and hypothalamic-pituitary-adrenal axis [2-7]. This has also been evidenced in humans using PET [8]. In the case of painful needling, afferent spinal gating and diffuse noxious inhibitory control (DNIC) may provide short-term analgesia [9].

However, these are not the only ways acupuncture may exert effects on the body. Interestingly, even though fMRI investigations have found greater subcortical responses for insertive acupuncture vs. non-insertive acupressure-like tapping they have shown stronger S1 response to the latter [10-13]. Recent neuroimaging data also suggest that the regular afferent stimulation 
provided by acupuncture may affect neuroplasticity in S1 cortex $[14,15]$. Thus, further investigation of the cortical signatures for electroacupuncture (EA) as well as acupressure stimulation would be useful. Since S1 neural activity occurs on a millisecond timescale, magnetoencephalography (MEG) may be used to non-invasively study these cortical responses.

In the present study, we used MEG to spatiotemporally map somatosensory cortical response to different types of acupuncture, electroacupuncture (EA) and acupressure (AP). Forearm acupoints were chosen based on ease of access and because MEG is biased towards superficial brain activity [16] making them easier to localize than leg acupoints. Importantly, we sought to mimic clinical acupuncture intervention procedures as much as possible. To do this we intentionally lacked control for attention and did not utilize concurrent sensory (i.e. non-EA or non-AP) stimuli. Furthermore, both EA and AP were given with clinically relevant parameters, i.e. $2 \mathrm{~Hz}$ stimulus rate and $>10 \mathrm{~min}$. duration. Finally, since acupoint specificity remains debatable [17] and may be exemplified predominantly as differences in somatotopic localization, we tested EA and AP at the same forearm acupoints.

\section{Results}

In the present study 16 healthy, right handed, subjects were given EA and non-invasive AP each at a low frequency $(2 \mathrm{~Hz}$ ) for 15 minutes while MEG was recorded.

\section{SI Sources and Evoked Timecourse Modulation}

For both EA and AP, the primary sources of MEG activity in each subject, localized to the contralateral primary somatosensory (SI) cortex, roughly area 3b (Figure 1a). EA/AP sources neighbored one another with AP sources mapping slightly dorsal to EA in most subjects. The average response for 15 minutes of EA stimulation (Figure 1b; black time-courses) first peaked at $20.6 \mathrm{~ms}$ poststimulus and then at 32.9 ms (black arrows). The spatiotemporal characteristics of these responses were similar to the M20 and M30 components evoked by median nerve stimulation $[18,19]$. For AP, initial peaks $25.5 \mathrm{~ms}$ and 38.5 ms shown (black arrows) were similar in orientation but delayed compared to EA. Both conditions also demonstrated peaks at $\sim 50 \mathrm{~ms}$ and $\sim 65 \mathrm{~ms}$, with those for AP again slightly delayed. Wide, long-latency, peaks occurred for both stimuli; at $120.2 \mathrm{~ms}$ for EA and 129.4 ms for AP. To determine if/how evoked SI responses were modulated during both 15 minute runs of EA and AP stimulation, individual data were divided into three, 5 minute sub-averages (Figure 1b; blue, red and green time-courses). These sub-averages showed attenuation of peak-to-peak amplitude for progressively later trials. Significant decreases in peak amplitude
(Figure 1c) following 5 minutes of stimulation (red (*) paired t-tests, $\mathrm{df}(15), \mathrm{p}<0.01)$ were found for EA at 30 and $50 \mathrm{~ms}$ and for AP at 50 and $130 \mathrm{~ms}$.

\section{Oscillatory Responses during EA and AP Stimulation}

Time frequency response (TFR) analysis demonstrated early and late modulation of oscillatory activity in S1 (Figure 2a). Early response included low gamma ( 30-50 $\mathrm{Hz}, \gamma)$ ERS from $\sim 20-70 \mathrm{~ms}$ post-stimulus and theta $(\sim 6-8 \mathrm{~Hz}, \theta)$ ERS at $\sim 50-200 \mathrm{~ms}$. This was followed by a prolonged decrease in mu frequency power, centered at alpha $(\sim 8-13 \mathrm{~Hz}, \alpha)$ and beta $(\sim 15-30 \mathrm{~Hz}, \beta)$ at $\sim 100$ $300 \mathrm{~ms}$ post-stimulus. No significant differences were found between EA and AP in any of these ranges (paired t-test, $\mathrm{p}<.01$ ).

To determine how/if oscillatory response changed over time, EA and AP data were segmented into fifteen separate averages, each 1 minute in length. The relative power between 100-300 ms post-stimulus for each minute and frequency band was assessed. Interestingly, over the course of EA the magnitude of beta ERD appeared to decrease (Figure 2b). A regression and goodness of fit test was performed, demonstrating a linear trend for EA $(\mathrm{R}=0.62$ and $\mathrm{p}=0.01)$. However for $\mathrm{AP}$, no significant linear trend was found $(R=0.35$ and $p=0.19)$. These same calculations were performed for the alpha, theta and gamma band (results available upon request) but no trends at the same significance level were seen. However, when comparing only the first and last minute, there appears to be some decrease in beta ERD for AP also (paired t-test $\mathrm{df}(15), \mathrm{p}<0.03)$. This reduction appears qualitatively as rapid reduction in induced response during the first 3-4 minutes of stimulation with no significant trend during later minutes. For EA, this same comparison was just under significance (paired t-test $\mathrm{df}(15), \mathrm{p}<0.07)$.

\section{Discussion}

In the present experiment we sought to spatiotemporally map somatosensory response to different types of acupuncture, EA and AP.

\section{Basic Response to EA and AP}

EA and AP responses most consistently mapped to contralateral SI (Figure 1a). However, AP produced a smaller/ broader M20 and slightly delayed M30 like responses (Figure 1b). Thus, with EA, underlying skin receptors/afferents were recruited simultaneously, while gradual skin indentation during AP likely produced temporal dispersion of afferent sensory signals. EEG studies have found that early SEPs (< $40 \mathrm{~ms})$ [20] and SEFs [21] to mechanical stimulation are often delayed and less pronounced than those from electrical stimulation. Furthermore, AP evoked stronger brain response than EA, particularly at long 


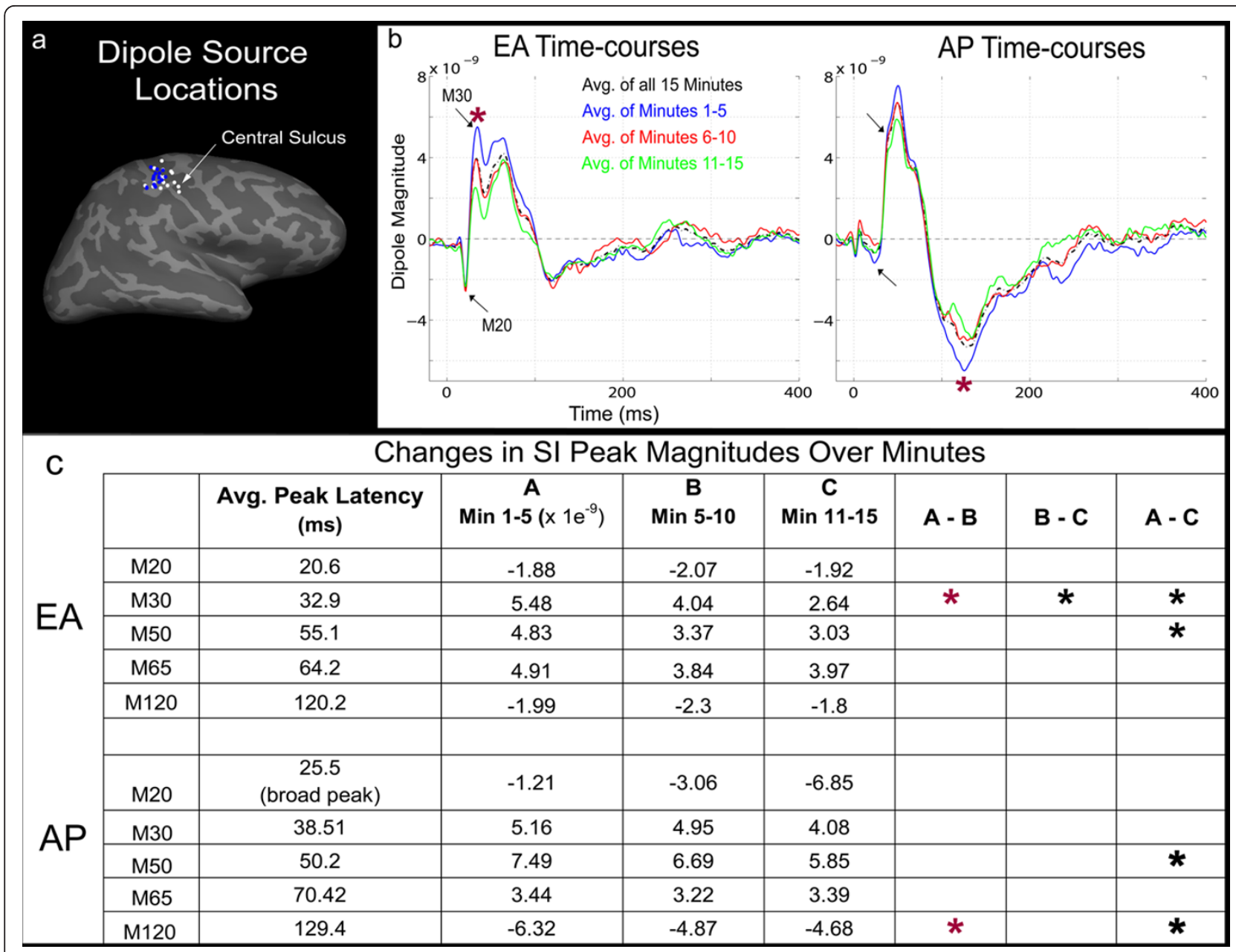

Figure 1 Evoked S1 responses to Electroacupuncture (EA) and Acupressure (AP): (a) For all subjects, the primary sources localized to the contralateral SI for EA (white dots) and AP (blue dots). Sources are shown as closest points on the cortex reconstructed from subjects' individual MRIs and morphed to an average inflated brain surface (sulci/gyri are dark/light gray respectively). (b) Average time-courses for all trials of EA and AP (black, dashed lines) demonstrated analogous M20, M30, M50/M60 and M120 (those of AP being slightly delayed). Five minute subaverages (blue, red and green lines) show attenuation of peak-to-peak amplitude over the course of the run. (c) Peaks demonstrating significant decreases $(p<0.01$ ) following 5 minutes of stimulation are marked by a red "**" For EA this was the M30 peaks and for AP the 130 ms peak. Significant differences occurring after > 10 minutes stimulation were seen for the M30 and M50 with EA and with the M50 and M120 with AP.

latencies (> $80 \mathrm{~ms}$ ). This may be explained by differences in the number and/or type of somatosensory fibers recruited. Although, both stimuli were carried at least in part by fast $A \beta$ sensory fibers, it is possible that the relatively larger surface area of the blunt acupressure needle tip activated more superficial sensory fibers than did EA. Differences in the magnitude of brain response to acupuncture vs. non-invasive control stimulation have also been noted in fMRI studies [10,13].

Time frequency response for EA (and AP) demonstrated early onset ERS at $\sim 20-70 \mathrm{~ms}$ in the gamma range (Figure 2a). Previous work utilizing visual tasks suggests that early gamma activity may reflect "stimulus selection" or local "binding" of attributes related to initial stimulus perception [22]. Similarly, the early latency of gamma activity in this study, suggests it may support stimulus selection in SI cortex. Theta ERD which began early ( $\sim 50 \mathrm{~ms}$ ) but continued into longer latencies $(\sim 200 \mathrm{~ms})$ may support general aspects of stimulus prediction [23]. Prolonged mu ERD 100-300 $\mathrm{ms}$, as in other tactile MEG studies, is consistent with the presence of afferent somatosensory input [24,25]. To understand if/how brain activity is modulated during the course of acupuncture and acupressure we assessed changes in the EA and AP response over the course of 15 minutes.

\section{Changes SI activity during EA and AP}

Cortical activity is thought to be in a constant state of use dependant fluctuation which may exist with or 


\section{Average Time Frequency Responses for a Electroacupuncture (EA) and Acupressure (AP)}
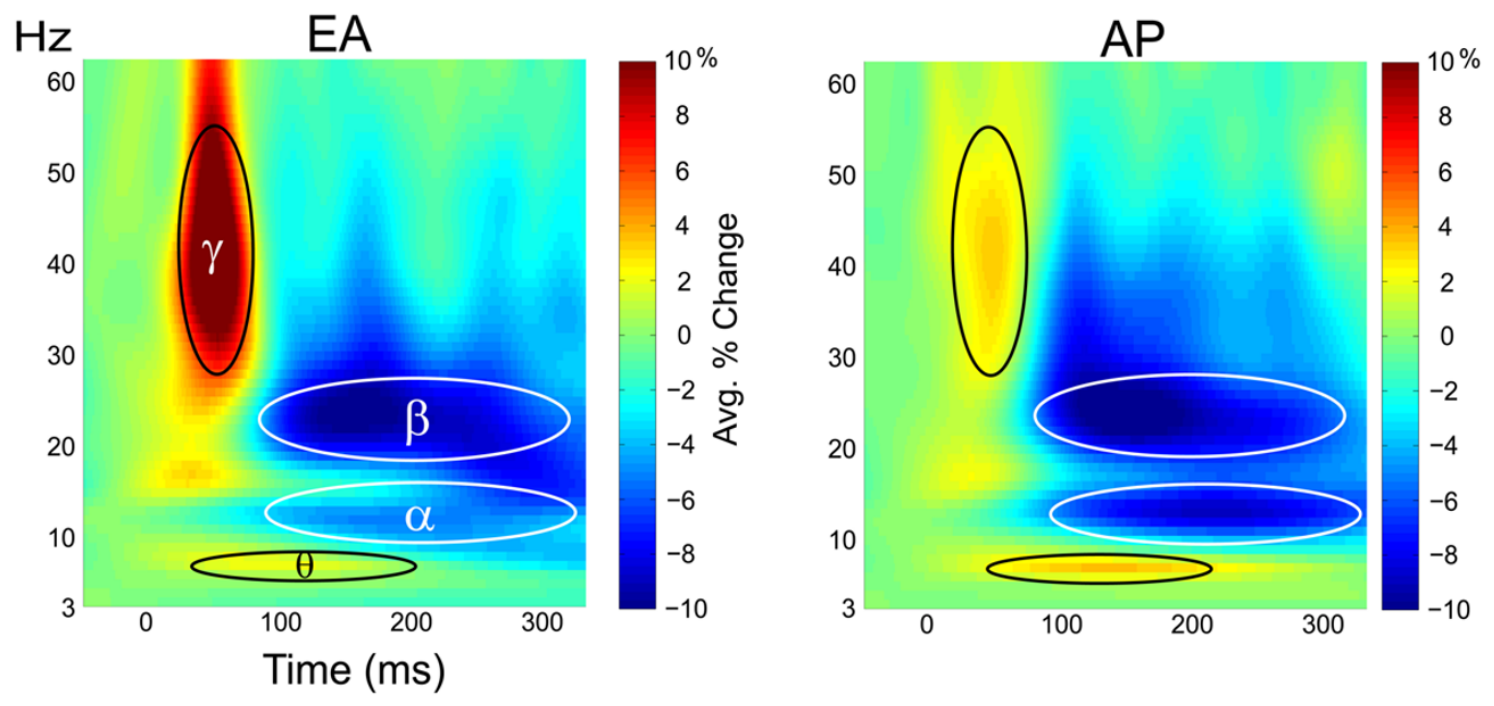

b

Changes in Beta Power at 100-300ms post-stimulus, over 15 minutes
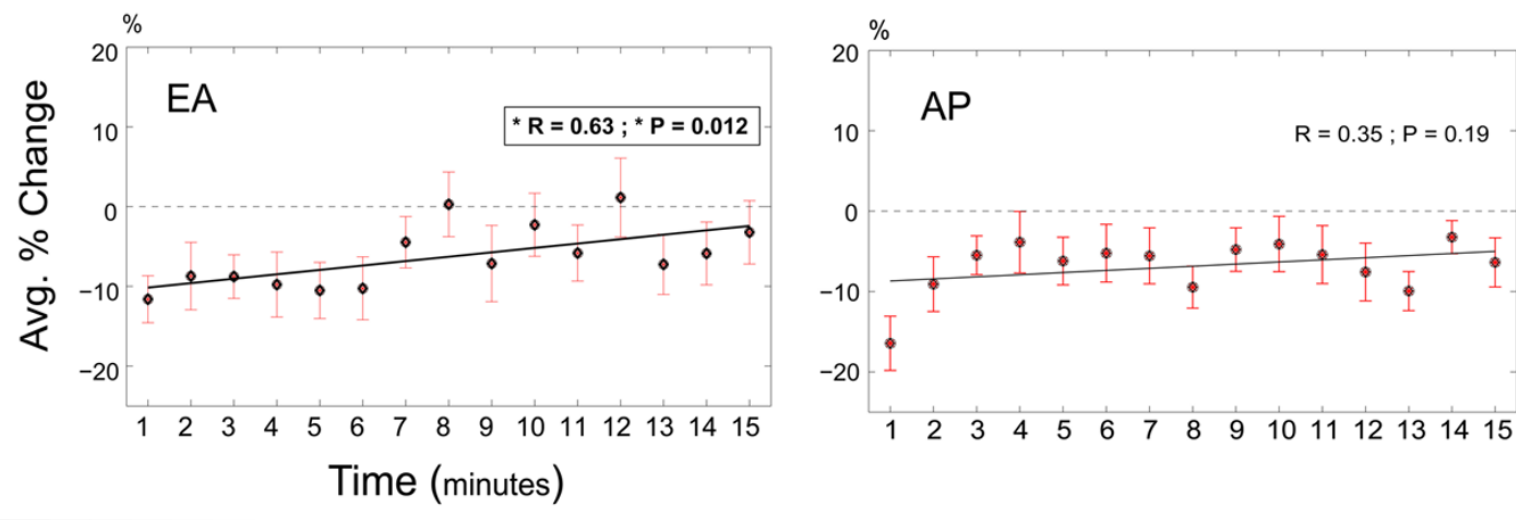

Figure 2 S1 Oscillatory Activity: (a) Post-stimulus time-frequency representations showed an early $20-70$ ms low-gamma ( $\boldsymbol{\gamma}$ ) ERS. This was followed by a prolonged ERD in alpha $(\boldsymbol{\alpha})$ and beta $(\boldsymbol{\beta})$ power at $\sim 100-300$ ms post-stimulus. There was a simultaneous theta $(\boldsymbol{\theta})$ ERS at $~ 50-200$ ms. (b) Beta ERD at 100-300 ms was calculated for each minute of stimulation and demonstrated a trend for decrease over the course of EA stimulation. Similar calculations were made for other frequency bands but did not show significance at the same $p<0.01$ level.

without higher order processes such as attention [26]. In our experiment, brain activity to EA and AP changed over many minutes, indicating habituation or conditioning of response.. Although, the first cortical response (M20) maintained a consistent amplitude, evoked responses at $>30 \mathrm{~ms}$ post-stimulus were significantly attenuated after 5 minutes of EA (Figure $1 \mathrm{~b}$ ). This was accompanied by a gradual decrease in beta ERD at $\sim 100-300 \mathrm{~ms}$ post-stimulus (Figure $2 \mathrm{~b}$ ) but not in the theta, alpha or gamma frequency bands (analysis available on request). Suggesting that in the domain of sensory conditioning, local beta band activity may be most important.

Furthermore, the earliest S1 evoked response (M20) represents bottom-up signals propagating from layer 4 (the primary site of thalamic afferent input) to layers $2 /$ 3 in cortex [27-30]. The post-stimulus latency of repetition induced decreases (i.e. occurring after $20 \mathrm{~ms}$ ) suggests they may be linked to top-down cortical mechanisms including decreased attention [18,31]. It should be noted that, in the present experiment we aimed to mimic a clinical acupuncture setting by 
maintaining continuous ( $\geq 10$ min.) acupoint stimulation and not actively engaging subjects in a concurrent distractor task. Similarly, mu ERD is also attenuated when subjects are less attentive to stimulus events [32-34]. Here, repetition induced decreases similar to those seen in somatosensory attentional paradigms likely reflects stimulus recognition and possibly, modification of somatosensory memories.

Recent data suggest acupuncture stimulation may induce beneficial cortical plasticity $[14,35]$ in neuropathic states. For example, following five-weeks of acupuncture treatment on the affected arm, carpal tunnel syndrome patients demonstrated clinical improvement (decreased parasthesias) and less S1 fMRI hyperactivation compared with pretreatment baseline [15]. Specifically it is hypothesized that the regular afferent input provided by acupuncture acts as a conditioning stimulus to counteract local symptoms of spontaneous activity occurring with parasthesias. It is well known that peripheral nerve lesions induce cortical reorganization [36-38] which may correlate with symptoms of hyperalgesia and pain [39]. However, such maladaptive plasticity may be reduced (or prevented) with therapy involving sensorimotor and/or visual feedback [40,41]. However, even in the absence of injury, somatosensory conditioning in the form of electrotherapy may help counteract normal age related sensorimotor decline [42].

In the electrophysiological domain, somatosensory conditioning may be marked not only by decreased ERF response but by changes in S1 beta activity over time. However, this is highly speculative and data from clinical populations is required. Although exposure related changes are also seen for AP the timing of evoked responses and the lack of change in the spectral domain suggests that a more regulated and sharper stimulus (EA) compared to a temporally diffuse mechanical stimulus (AP). Thus, although both may prove useful somatosensory conditioning stimuli, it is possible that EA may prove to be a better conditioning stimulus for neuropathic conditions where maladaptive central plasticity may be maintained by diffuse unregulated (spontaneous) afference - i.e. paresthesias in CTS. However, this is again speculative and requires testing in clinical populations.

\section{Conclusion}

We used MEG to map somatosensory brain response during 15 minutes of electroacupuncture and acupressure. ERF's to EA and AP most consistently localized to contralateral SI. However, AP differed in its temporal dynamics showing delayed response peaks, consistent with mechanical stimulation. Both EA and AP demonstrated significantly decreased response amplitudes, following five minutes of stimulation. However, the latency of these decreases occurred much earlier in EA ( 30 ms post-stimulus) than AP (> $100 \mathrm{~ms}$ ). Time-frequency responses demonstrated early onset, event related synchronization (ERS), within the gamma band at $\sim 70-130$ $\mathrm{ms}$ and the theta band at $\sim 50-200 \mathrm{~ms}$ post-stimulus. A prolonged event related desynchronization (ERD) of alpha and beta power occurred at $\sim 100-300 \mathrm{~ms}$ post-stimulus. There was decreased beta ERD at $\sim 100-300 \mathrm{~ms}$ over the course of EA, but not with AP. The precise timing provided by EA stimulation supports its role as a conditioning stimulus which may be used to affect maladaptive neuroplasticity.

\section{Methods}

\section{Subjects and Experimental Paradigm}

Imaging data were collected from 16 healthy subjects between 20-44 years of age. This study was in compliance with the Helsinki Declaration. All subjects gave written consent and were screened to assure their safety and compatibility for MEG and MRI recordings. During the MEG experiment subjects were seated with their head in the dewar and instructed to fixate on a centrally presented "+" sign. Both EA and AP consisted of 15 minutes continuous low-frequency $(2 \mathrm{~Hz})$ stimulation given on the left medial forearm at acupoint PC-6 (pericardium-6, neiguan). Forearm acupoints were used primarily based on ease of access and because MEG is biased towards superficial brain activity. Thus, SI responses for points on the forearm are more accurately mapped with MEG than those of the leg which are located medially in the brain [16]. All acupuncture was performed by the same licensed acupuncturist and the order of EA and AP runs was randomized across subjects. During each 10 minute rest run there was no acupuncture intervention and subjects were required to sit quietly. Rest runs were used to reduce residual sensations between acupuncture runs. Subjects wore earplugs throughout the experiment to attenuate any sounds heard from outside the MEG room or from stimulation equipment.

\section{Acupuncture Procedures}

Participants were told they would receive "two different types" of acupuncture and were prevented from viewing insertion and stimulation procedures through the use of an opaque screen. During the experiment subjects wore a plastic brace on their forearm to prevent fist clenching and excessive hand movement. A rectangular opening over the ventral forearm provided access to acupoints. The EA run involved needle insertion and manipulation, to elicit deqi ${ }^{1}$ sensation, after which electrical current was delivered. Current amplitude was set to the level at which subjects indicated feeling a "strong but not painful" sensation. Current was delivered as a monophasic 
rectangular, constant-current pulse (pulse width: $0.2 \mathrm{~ms}$ at $2 \mathrm{~Hz}$ ) using a GRASS stimulator (S88 Dual Output Square Pulse Stimulator, Grass Telefactor, West Warwick, RI). To most closely match active stimulation in the EA run, the AP procedure involved AP insertion followed by stimulation. During AP insertion, subjects were palpated near the acupoint to mimic acupoint localization (as with EA). Then needle insertion and manipulation was simulated with a previously validated technique [43] using a wooden toothpick positioned at the acupoint with a guide tube. The toothpick was manipulated and subjects were asked what sensations they felt and if there was any pain. During this time the piezo-stimulator tip was lowered over the acupoint. The stimulation consisted of a $2 \mathrm{~Hz}$ mechanical pecking to mimic EA frequency. To do this the plastic arm brace was equipped with a piezo-electric cantilever beam (Piezo Bender Q-503B, Piezo Systems, Cambridge, MA). The device was battery powered and controlled with National Instruments (NI) Labview program in combination with the 6100 DAQ card (NI) located in a laptop with Labview software. The digital signal was converted with a D/A converter and amplified (Low Cost Linear Amplifier, Piezo Systems Inc.) prior to reaching the piezo. The stimulus waveform was a single lobe from a $100 \mathrm{~Hz}$ half-sine wave (pulse width $5 \mathrm{~ms}$ ). A similar "tapping" procedure has been conducted manually in acupuncture fMRI studies $[13,44]$ and was also chosen here as it approximates control procedures used in many clinical trials

\section{MEG Data Collection}

MEG signals were recorded with a 306-channel Vectorview MEG system (Elekta Neuromag Oy, Helsinki, Finland) housed in a custom six-layer magnetically shielded room [45]. The head position was monitored during the measurement using head position indicator coils (HPI). Locations on the subject's scalp surface and the HPI coils were digitized using a Polhemus FastTrak digitizer to allow for accurate alignment of the MEG sensor array with the subjects MRI scan. Importantly, to help minimize head motion, foam padding was used and subjects were reminded to avoid slouching and remain still. The acquisition bandwidth was 0$400 \mathrm{~Hz}$ with a 1200 samples/sec digitization rate. The subject's electrocardiogram (ECG) and electro-occulogram (EOG) were recorded simultaneously to control for and if necessary remove influence from physiological noise sources such as heart beat, eye blinks and eye saccading. The raw data were further processed using the signal space separation (MaxFilter, Elekta Neuromag Oy, Helsinki, Finland) to reduce the influence of magnetic fields originating from outside the subjects head.

\section{Structural MRI Data Collection and Cortical Surface Reconstruction}

Each subject underwent an MRI scan which was coregistered with the MEG data. The anatomical MRI was used for creation of boundary element models and visualization of the cortical surface anatomy. Each subject was scanned in a Siemens Avanto 1.5 T MRI (Siemens Medical, Erlangen, Germany). Two highresolution MPRAGE $(256 \times 192$ matrix, $256 \mathrm{~mm}$ FOV,128 slices, $1.33 \mathrm{~mm}$ slice thickness, TE $=3.31 \mathrm{~ms}$, $\mathrm{TR}=2730 \mathrm{~ms}$, $\mathrm{TI}=1000 \mathrm{~ms}$, flip $=7 \mathrm{deg}$ ) volumes (motion corrected and averaged offline) and a multiecho 3D-FLASH scan $(256 \times 192$ matrix, $256 \mathrm{~mm}$ FOV, 128 slices, $1.33 \mathrm{~mm}$ slice thickness, $\mathrm{TE}=1.85, \mathrm{TR}=20$ ms, 3 echoes, echo spacing $=100 \mu \mathrm{s}$, flip $=5 \mathrm{deg}$ ) were acquired.

A geometrical representation of the cortical surface of each subject was obtained using procedures described previously $[46,47]$. First, using each subject's high-resolution 3-D, T1-weighted structural image, the cortical white matter was segmented, and the estimated border between gray and white matter was tessellated, providing a topologically correct representation of the surface with about 150,000 vertices per hemisphere. The folded surface tessellation was "inflated" in order to unfold cortical sulci, thereby providing a convenient format for visualizing MEG sources. The reconstructed surface for each subject was morphed into an average spherical representation, optimally aligning sulcal and gyral features across subjects while minimizing metric distortions and shear [47]. Compared to volumetric morphing into Talairach [48] space, this method has been found to provide better alignment across subjects of functional activation in a verbal task [49] and allows direct localization to regular gyri.

\section{MEG Data Processing}

For evoked time-course analysis, data were low-pass-filtered at $150 \mathrm{~Hz}$ and separate averages of each condition were constructed for all subjects. Trials were rejected from analysis based on amplitude criteria supplemented by visual inspection for contamination by artifacts (identified as peak-to-peak amplitude $(>3,000 \mathrm{fT} / \mathrm{cm}$ in any channel) or eye blinks ( $>250 \mathrm{mV}$ in the EOG electrode). For each subject, S1 dipole models were fit for the 20/30 ms response for acupuncture conditions using the xfit software (Elekta Neuromag Oy, Helsinki, Finland). The location of the dipoles was projected to the cortical surface of each subject using individual MRI scans and then mapped to an average cortical surface via the spherical atlas provided by FreeSurfer $[46,47]$. The dipole locations for all subjects on the average cortical surface are shown in Figure 1a. Spatial filters corresponding to the P30 dipoles were utilized to generate 
continuous raw waveforms for each subject and condition. All subsequent analysis was performed on these waveforms. To obtain the evoked time-courses waveforms were averaged on stimulus triggers. To visualize changes in the evoked response over the course of the experiment, data were subdivided into 3 sequential windows of 5 minutes averages (Figure 1b). Significant differences between these 5 minute averages were assessed with a one-tailed paired $t$-test comparing the mean dipolar moment at peaks and during specific post-stimulus time intervals (Figure 1c).

Time-frequency response (TFR) analysis of the S1 source waveforms was performed to investigate (Figure 2a) induced activity during acupuncture stimulation. Induced activity describes a change in the ongoing or endogenous oscillatory activity of the brain; this activity is not phase-locked to the stimulus and cannot be seen with simple event-related analyses. In order to calculate a time-frequency distribution for these MEG data we employed a continuous wavelet transform using complex Morlet wavelets [50]. Since our study utilized very short inter-stimulus intervals $(500 \mathrm{~ms})$, continuous raw waveforms were wavelet transformed prior to separation into trials. This was done to prevent edge artifact contamination in the lower frequency spectrum. An average "evoked" TFR was calculated for both EA and AP conditions then subtracted from each individual trial prior to averaging for creation of the induced TFRs. The percentage change relative to the baseline mean was calculated for each frequency individually, to determine the level of ERD (event related desynchronization) and ERS (event related synchronization) [51]. To confirm that the observed induced changes were not simply the effect of a slow trending and to determine if there were timedependent changes in the induced response, the TFR analysis was performed on sequential windows of $1 \mathrm{~min}$ ute duration. The average percent-change values across subjects for each minute and condition are shown for a time-frequency ROI in Figure $2 b$.

\section{Endnotes}

${ }^{1}$ Translates as "obtaining qi" and traditionally refers to sensations (e.g. soreness, aching, warmth, etc.) that have been used to indicate accurate localization of an acupoint.

\footnotetext{
Acknowledgements

We would like to thank NIH NCCAM for funding support K01AT004481 (Dhond); K01AT002166, R01AT004714 (Napadow); P01AT002048 (Rosen). We also acknowledge NCRR (P41RR14075), and the Mental Illness and Neuroscience Discovery (MIND) Institute. We thank: Yoshio Okada, Claudio Babiloni, Elina Pihko, Hubert Dinse, Alfons Schnitzler, Aziz Ashgar, Hugh Macpherson, Bruce Rosen, Ted Kaptchuk (K24AT004095) and two anonymous reviewers for helpful comments and/or discussion on this manuscript. We also thank Simon Sigalovsky for assistance constructing the
}

acupressure device. The content of this article is solely the responsibility of the authors and does not necessarily represent the official views of our funding agencies.

\section{Author details}

${ }^{1}$ Harvard Medical School, Martinos Center for Biomedical Imaging, Charlestown, MA 02129 USA. ${ }^{2}$ Logan College of Chiropractic, Department of Radiology, Chesterfield, MO 63017 USA.

\section{Authors' contributions}

TW generated analysis tools and contributed significantly to data acquisition analysis and manuscript writing. VN participated in study design, manuscript editing and was the acupuncturist for the study. NK participated in study design and manuscript editing. MV was critical in statistical analysis of data. $\mathrm{MH}$ generated analysis tools and participated in manuscript editing. RD was essential to all aspects of study design, data acquisition, analysis and

manuscript writing. All authors read and approved the final manuscript.

Received: 26 May 2011 Accepted: 27 July 2011 Published: 27 July 2011

\section{References}

1. Dhond RP, Kettner N, Napadow V: Do the neural correlates of acupuncture and placebo effects differ? Pain 2007, 128(1-2):8-12.

2. Pomeranz B, Chiu D: Naloxone blockade of acupuncture analgesia: endorphin implicated. Life Sci 1976, 19(11):1757-1762.

3. Han JS, Terenius L: Neurochemical basis of acupuncture analgesia. Annu Rev Pharmacol Toxicol 1982, 22:193-220.

4. Takeshige C: Mechanisms of Acupuncture Analgesia Produced by Low Frequency Electrical Stimulation of Acupuncture Points. In Clinical Acupuncture: Scientific Basis. Edited by: Stux G, Hammerschlag R. Berlin: Springer-Verlag; 2001:29-50.

5. Le Bars D, Villanueva L, Bouhassira D, Willer JC: Diffuse noxious inhibitory controls (DNIC) in animals and in man. Patol Fiziol Eksp Ter 1992, , 4: 55-65.

6. Le Bars D, Willer JC, De Broucker T: Morphine blocks descending pain inhibitory controls in humans. Pain 1992, 48(1):13-20.

7. White A: Neurophysiology of Acupuncture Analgesia. In Acupuncture: A Scientific Appraisal. Volume Chapter 3. Oxford: Butterworth-Heinemann; 1999.

8. Harris RE, Zubieta JK, Scott DJ, Napadow V, Gracely RH, Clauw DJ: Traditional Chinese acupuncture and placebo (sham) acupuncture are differentiated by their effects on mu-opioid receptors (MORs). Neurolmage 2009, 47(3):1077-1085.

9. Carlsson C: Acupuncture mechanisms for clinically relevant long-term effects-reconsideration and a hypothesis. Acupunct Med 2002, 20(23):82-99.

10. Hui KK, Liu J, Makris N, Gollub RL, Chen AJ, Moore Cl, Kennedy DN, Rosen BR, Kwong KK: Acupuncture modulates the limbic system and subcortical gray structures of the human brain: evidence from fMRI studies in normal subjects. Hum Brain Mapp 2000, 9(1):13-25.

11. Hui KK, Liu J, Marina O, Napadow V, Haselgrove C, Kwong KK, Kennedy DN, Makris N: The integrated response of the human cerebro-cerebellar and limbic systems to acupuncture stimulation at ST 36 as evidenced by fMRI. Neuroimage 2005, 27(3):479-496.

12. Napadow V, Dhond R, Park K, Kim J, Makris N, Kwong KK, Harris RE, Purdon PL, Kettner N, Hui KK: Time-variant fMRI activity in the brainstem and higher structures in response to acupuncture. Neurolmage 2009, 47(1):289-301.

13. Napadow V, Makris N, Liu J, Kettner NW, Kwong KK, Hui KK: Effects of electroacupuncture versus manual acupuncture on the human brain as measured by fMRI. Hum Brain Mapp 2005, 24(3):193-205.

14. Li G, Jack CR Jr, Yang ES: An fMRI study of somatosensory-implicated acupuncture points in stable somatosensory stroke patients. J Magn Reson Imaging 2006, 24(5):1018-1024.

15. Napadow V, Liu J, Li M, Kettner N, Ryan A, Kwong KK, Hui KK, Audette JF: Somatosensory cortical plasticity in carpal tunnel syndrome treated by acupuncture. Hum Brain Mapp 2007, 28(3):159-171.

16. Hamalainen M, Hari R: Magnetoencephalographic Characterization of Dynamic Brain Activation: Basic Principles and Methods of Data Collection and Source Analysis. In Brain Mapping: The Methods. Volume Ch 10.. 2 edition. Edited by: Toga AW, Mazziotta JC. San Diego: Academic Press; 2002 
17. Zhang H, Bian Z, Lin Z: Are acupoints specific for diseases? A systematic review of the randomized controlled trials with sham acupuncture controls. Chin Med 2010, 5:1.

18. Mauguiere F, Merlet I, Forss N, Vanni S, Jousmaki V, Adeleine P, Hari R: Activation of a distributed somatosensory cortical network in the human brain: A dipole modelling study of magnetic fields evoked by median nerve stimulation. Part II: Effects of stimulus rate, attention and stimulus detection. Electroencephalogr Clin Neurophysiol 1997, 104(4):290-295.

19. Mauquiere F, Merlet I, Forss N, Vanni S, Jousmaki V, Adeleine P, Hari R: Activation of a distributed somatosensory cortical network in the human brain. A dipole modelling study of magnetic fields evoked by median nerve stimulation. Part I: Location and activation timing of SEF sources. Electroencephalogr Clin Neurophysiol 1997, 104(4):281-289.

20. Kakigi $R$, Shibasaki $H$ : Scalp topography of the short latency somatosensory evoked potentials following posterior tibial nerve stimulation in man. Electroencephalogr Clin Neurophysiol 1983, 56(5):430-437.

21. Forss N, Salmelin R, Hari R: Comparison of somatosensory evoked fields to airpuff and electric stimuli. Electroencephalogr Clin Neurophysiol 1994, 92(6):510-517.

22. Fries $P$, Roelfsema PR, Engel AK, Konig P, Singer W: Synchronization of oscillatory responses in visual cortex correlates with perception in interocular rivalry. Proc Natl Acad Sci USA 1997, 94(23):12699-12704.

23. Babiloni C, Brancucci A, Vecchio F, Arendt-Nielsen L, Chen AC, Rossini PM: Anticipation of somatosensory and motor events increases centroparietal functional coupling: an EEG coherence study. Clin Neurophysiol 2006, 117(5):1000-1008.

24. Hari R, Salmelin R, Makela J, Salenius S, Helle M: Magnetoencephalographic cortical rhythms. IntJPsychophysiol 1997, 26(1-3):51-62.

25. Schnitzler A, Salenius S, Salmelin R, Jousmaki V, Hari R: Involvement of primary motor cortex in motor imagery: a neuromagnetic study. Neuroimage 1997, 6(3):201-208.

26. Dinse HR, Merzenich M: Adaptation of Inputs in the somatosensory system. In Perceptual Learning. Edited by: Fahle M, Poggio T. Cambridge, USA: MIT Press; 2002:19-42.

27. Wikstrom H, Huttunen J, Korvenoja A, Virtanen J, Salonen O, Aronen H, IImoniemi RJ: Effects of interstimulus interval on somatosensory evoked magnetic fields (SEFs): a hypothesis concerning SEF generation at the primary sensorimotor cortex. Electroencephalogr Clin Neurophysiol 1996, 100(6):479-487.

28. Ikeda H, Wang Y, Okada YC: Origins of the somatic N20 and highfrequency oscillations evoked by trigeminal stimulation in the piglets. Clin Neurophysiol 2005, 116(4):827-841.

29. Allison T, Wood CC, McCarthy G, Spencer DD: Cortical somatosensory evoked potentials. II. Effects of excision of somatosensory or motor cortex in humans and monkeys. J Neurophysiol 1991, 66(1):64-82.

30. Tiihonen J, Hari R, Hamalainen M: Early deflections of cerebral magnetic responses to median nerve stimulation. Electroencephalogr Clin Neurophysiol 1989, 74(4):290-296.

31. Desmedt JE, Tomberg C: Mapping early somatosensory evoked potentials in selective attention: critical evaluation of control conditions used for titrating by difference the cognitive P30, P40, P100 and N140. ElectroencephClinNeurophysiol 1989, 74:321-346.

32. Dockstader C, Cheyne D, Tannock R: Cortical dynamics of selective attention to somatosensory events. Neurolmage 2009.

33. Bauer $M$, Oostenveld $R$, Peeters $M$, Fries $P$ : Tactile spatial attention enhances gamma-band activity in somatosensory cortex and reduces low-frequency activity in parieto-occipital areas. J Neurosci 2006, 26(2):490-501.

34. Bardouille T, Picton TW, Ross B: Attention modulates beta oscillations during prolonged tactile stimulation. Eur J Neurosci 2010, 31(4):761-769.

35. Lo YL, Cui SL, Fook-Chong S: The effect of acupuncture on motor cortex excitability and plasticity. Neurosci Lett 2005, 384(1-2):145-149.

36. Merzenich MM, Nelson RJ, Stryker MP, Cynader MS, Schoppmann A, Zook JM: Somatosensory cortical map changes following digit amputation in adult monkeys. J Comp Neurol 1984, 224(4):591-605.

37. Ramachandran VS, Stewart M, Rogers-Ramachandran DC: Perceptual correlates of massive cortical reorganization. Neurorep 1992, 3(7):583-586.

38. Elbert T, Flor H, Birbaumer N, Knecht S, Hampson S, Larbig W, Taub E: Extensive reorganization of the somatosensory cortex in adult humans after nervous system injury. Neurorep 1994, 5(18):2593-2597.
39. Flor H, Elbert T, Knecht S, Wienbruch C, Pantev C, Birbaumer N, Larbig W, Taub E: Phantom-limb pain as a perceptual correlate of cortical reorganization following arm amputation. Nature 1995, 375(6531):482-484.

40. Lotze M, Grodd W, Birbaumer N, Erb M, Huse E, Flor H: Does use of a myoelectric prosthesis prevent cortical reorganization and phantom limb pain? Nat Neurosci 1999, 2(6):501-502.

41. Ramachandran VS: Plasticity and functional recovery in neurology. Clin Med 2005, 5(4):368-373.

42. Kalisch T, Tegenthoff M, Dinse HR: Improvement of sensorimotor functions in old age by passive sensory stimulation. Clin Interv Aging 2008, 3(4):673-690

43. Sherman KJ, Hogeboom CJ, Cherkin DC, Deyo RA: Description and validation of a noninvasive placebo acupuncture procedure. J Altern Complement Med 2002, 8(1):11-19.

44. Dhond RP, Yeh C, Park K, Kettner N, Napadow V: Acupuncture modulates resting state connectivity in default and sensorimotor brain networks. Pain 2008, 136(3):407-418.

45. Cohen D, Schlapfer U, Ahlfors S, Hamalainen M, Halgren E: New Six-Layer Magnetically-Shielded Room for MEG. In 13th International Conference on Biomagnetism. Edited by: Nowak H, Haueisen J, Geisler F, Huonker R Verlag. Jena, Germany; 2002:919.

46. Dale AM, Fischl B, Sereno Ml: Cortical surface-based analysis I: Segmentation and surface reconstruction. Neurolmage 1999, 9(2):179-194.

47. Fischl B, Sereno MI, Dale AM: Cortical surface-based analysis. II: Inflation, flattening, and a surface-based coordinate system. Neuroimage 1999, 9(2):195-207.

48. Collins DL, Neelin P, Peters TM, Evans AC: Data in Standardized Talairach Space. JComputAssistTomogr 1994, 18(2):292-205.

49. Fischl B, Sereno MI, Tootell RB, Dale AM: High-resolution intersubject averaging and a coordinate system for the cortical surface. Hum Brain Mapp 1999, 8(4):272-284.

50. Goupillaud P, Grossman A, Morlet J: Cycle-octave and related transforms in seismic signal analysis. Geoexploration 1984, 23:85.

51. Graimann B, Huggins JE, Levine SP, Pfurtscheller G: Visualization of significant ERD/ERS patterns in multichannel EEG and ECoG data. Clin Neurophysiol 2002, 113(1):43-47.

doi:10.1186/1471-2202-12-73

Cite this article as: Witzel et al:: Differences in cortical response to acupressure and electroacupuncture stimuli. BMC Neuroscience 2011 $12: 73$.

\section{Submit your next manuscript to BioMed Central and take full advantage of:}

- Convenient online submission

- Thorough peer review

- No space constraints or color figure charges

- Immediate publication on acceptance

- Inclusion in PubMed, CAS, Scopus and Google Scholar

- Research which is freely available for redistribution 